\title{
CONTROLLABILITY OF PARABOLIC EQUATIONS BY THE FLATNESS APPROACH
}

\author{
by
}

Philippe Martin, Lionel Rosier \& Pierre Rouchon

\begin{abstract}
We consider linear one-dimensional parabolic equations with space dependent coefficients that are only measurable and that may be degenerate or singular. We prove the null controllability with one boundary control by following the flatness approach, which provides explicitly the control and the associated trajectory as series. As an application, we consider the heat equation with a discontinuous coefficient in the principal part. The note ends with a numerical experiment which demonstrates the effectiveness of the method.
\end{abstract}

\section{Contents}

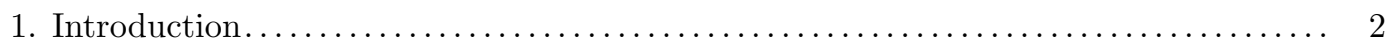

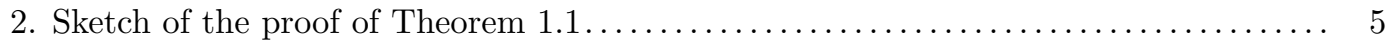

3. Numerical control of the 1-D heat equation with discontinuous coefficients....... 9

4. Numerical control of a degenerate 1 -D heat equation $\ldots \ldots \ldots \ldots \ldots \ldots \ldots \ldots \ldots \ldots 11$

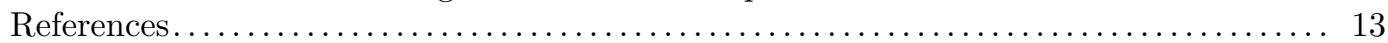

2000 Mathematics Subject Classification. - 93B05,93C20.

Key words and phrases. — degenerate parabolic equation; singular coefficient; null controllability, Gevrey functions; flatness. 


\section{Introduction}

The null controllability of parabolic equations has been extensively investigated since several decades. After the pioneering work in $[\mathbf{1 1}, \mathbf{1 5}, \mathbf{2 0}]$, mainly concerned with the one-dimensional case, there has been significant progress in the general $\mathrm{N}$-dimensional case $[\mathbf{1 3}, \mathbf{1 4}, \mathbf{1 9}]$ by using Carleman estimates. The more recent developments of the theory were concerned with discontinuous coefficients $[\mathbf{1}, \mathbf{2}]$, degenerate coefficients $[4, \mathbf{5}, \mathbf{6}, \mathbf{1 2}]$, or singular coefficients $[\mathbf{8}, \mathbf{1 0}]$.

In $[\mathbf{1}]$, the authors derived the null controllability of a linear one-dimensional parabolic equation with (essentially bounded) measurable coefficients. The method of proof combined the Lebeau-Robbiano approach [19] with some complex analytic arguments.

Here, we are concerned with the null controllability of the system

$$
\begin{aligned}
\left(a(x) u_{x}\right)_{x}+b(x) u_{x}+c(x) u-\rho(x) u_{t} & =0, \quad x \in(0,1), t \in(0, T), \\
\alpha_{0} u(0, t)+\beta_{0}\left(a u_{x}\right)(0, t) & =0, \quad t \in(0, T), \\
\alpha_{1} u(1, t)+\beta_{1}\left(a u_{x}\right)(1, t) & =h(t), \quad t \in(0, T), \\
u(x, 0) & =u_{0}(x), \quad x \in(0,1),
\end{aligned}
$$

where $\left(\alpha_{0}, \beta_{0}\right),\left(\alpha_{1}, \beta_{1}\right) \in \mathbb{R}^{2} \backslash\{(0,0)\}$ are given, $u_{0} \in L^{2}(0,1)$ is the initial state and $h \in L^{2}(0, T)$ is the control input.

The given functions $a, b, c, \rho$ will be assumed to fulfill the following conditions

$$
\begin{aligned}
& a(x)>0 \text { and } \rho(x)>0 \text { for a.e. } x \in(0,1), \\
& \left(\frac{1}{a}, \frac{b}{a}, c, \rho\right) \in\left[L^{1}(0,1)\right]^{4}, \\
& \exists K \geq 0, \quad \frac{c(x)}{\rho(x)} \leq K \text { for a.e. } x \in(0,1), \\
& \exists p \in(1, \infty], \quad a^{1-\frac{1}{p}} \rho \in L^{p}(0,1) .
\end{aligned}
$$

The assumptions (1.5)-(1.8) are clearly less restrictive than the assumptions from [1]:

$$
a, b, c, \rho \in L^{\infty}(0,1) \text { and } a(x)>\varepsilon, \rho(x)>\varepsilon>0 \text { for a.e. } x \in(0,1)
$$

for some $\varepsilon>0$.

Let us introduce some notations. Let $B$ be a Banach space with norm $\|\cdot\|_{B}$. For any $t_{1}<t_{2}$ and $s \geq 0$, we denote by $G^{s}\left(\left[t_{1}, t_{2}\right], B\right)$ the class of (Gevrey) functions $u \in C^{\infty}\left(\left[t_{1}, t_{2}\right], B\right)$ for which there exist positive constants $M, R$ such that

$$
\left\|u^{(p)}(t)\right\|_{B} \leq M \frac{p !^{s}}{R^{p}} \quad \forall t \in\left[t_{1}, t_{2}\right], \forall p \geq 0 .
$$

When $\left(B,\|\cdot\|_{B}\right)=(\mathbb{R},|\cdot|), G^{s}\left(\left[t_{1}, t_{2}\right], B\right)$ is merely denoted $G^{s}\left(\left[t_{1}, t_{2}\right]\right)$. Let

$$
L_{\rho}^{1}:=\left\{u:(0,1) \rightarrow \mathbb{R} ; \quad\|u\|_{L_{\rho}^{1}}:=\int_{0}^{1}|u(x)| \rho(x) d x<\infty\right\} .
$$

Note that $L^{2}(0,1) \subset L_{\rho}^{1}$ if $\rho \in L^{2}(0,1)$. The main result in this note is the following

Theorem 1.1. - Let the functions $a, b, c, \rho:(0,1) \rightarrow \mathbb{R}$ satisfy (1.5)-(1.8) for some numbers $K \geq 0$, $p \in(1, \infty]$. Let $\left(\alpha_{0}, \beta_{0}\right),\left(\alpha_{1}, \beta_{1}\right) \in \mathbb{R}^{2} \backslash\{(0,0)\}$ and $T>0$. Pick any $u_{0} \in L_{\rho}^{1}$ and any $s \in(1,2-1 / p)$. Then there exists a function $h \in G^{s}([0, T])$, that may be given explicitly as a series, such that the solution $u$ of (1.1)-(1.4) satisfies $u(., T)=0$. Moreover $u, a u_{x} \in G^{s}\left([\varepsilon, T], W^{1,1}(0,1)\right)$ for all $\varepsilon \in(0, T)$. 
Clearly, Theorem 1.1 can be applied to parabolic equations with discontinuous coefficients that may be degenerate or singular at a point (or more generally at a sequence of points). The proof of it is not based on the classical duality approach, in the sense that it does not rely on the proof of some observability inequality for the adjoint equation. It follows the flatness approach developed in $[\mathbf{1 6}, \mathbf{1 7}, \mathbf{1 8}, \mathbf{2 1}, \mathbf{2 2}$, $\mathbf{2 4}, \mathbf{2 5}, \mathbf{2 7}, \mathbf{2 8}, \mathbf{2 9}$ ]. This direct approach gives explicitly both the control and the trajectory as series, which leads to efficient numerical schemes by taking partial sums in the series [24]. The flatness approach was used in $[\mathbf{2 8}]$ to improve the results in $[\mathbf{1 1}]$ concerning the reachable states for the heat equation. It was also successfully applied to Schrödinger equation in [23]. Let us describe its main steps. In the first step, following [1], we show that after a series of changes of dependent/independent variables, system (1.1)-(1.4) can be put into the canonical form

$$
\begin{aligned}
u_{x x}-\rho(x) u_{t} & =0, \quad x \in(0,1), t \in(0, T), \\
\alpha_{0} u(0, t)+\beta_{0} u_{x}(0, t) & =0, \quad t \in(0, T), \\
\alpha_{1} u(1, t)+\beta_{1} u_{x}(1, t) & =h(t), \quad t \in(0, T), \\
u(x, 0) & =u_{0}(x), \quad x \in(0,1),
\end{aligned}
$$

where $\rho(x)>0$ a.e. in $(0,1)$ and $\rho \in L^{p}(0,1)$ with $p \in(1, \infty]$. In the second step, following $[\mathbf{2 1}, \mathbf{2 4}$, we seek $u$ in the form

$$
\begin{aligned}
& u(x, t)=\sum_{n \geq 0} e^{-\lambda_{n} t} e_{n}(x), \quad x \in(0,1), t \in[0, \tau], \\
& u(x, t)=\sum_{i \geq 0} y^{(i)}(t) g_{i}(x), \quad x \in(0,1), t \in[\tau, T],
\end{aligned}
$$

where $\tau \in(0, T)$ is any intermediate time; $e_{n}:(0,1) \rightarrow \mathbb{R}$ (resp. $\left.\lambda_{n} \in \mathbb{R}\right)$ denotes the $n^{\text {th }}$ eigenfunction (resp. eigenvalue) associated with (1.11)-(1.13) and satisfying $[\mathbf{1 6}, \mathbf{1 7}]$

$$
\begin{aligned}
-e_{n}^{\prime \prime} & =\lambda_{n} \rho e_{n}, \quad x \in(0,1) \\
\alpha_{0} e_{n}(0)+\beta_{0} e_{n}^{\prime}(0) & =0, \\
\alpha_{1} e_{n}(1)+\beta_{1} e_{n}^{\prime}(1) & =0,
\end{aligned}
$$

while $g_{i}:(0,1) \rightarrow \mathbb{R}$ is defined inductively as the solution to the Cauchy problem

$$
\begin{aligned}
g_{0}^{\prime \prime} & =0, \quad x \in(0,1) \\
\alpha_{0} g_{0}(0)+\beta_{0} g_{0}^{\prime}(0) & =0, \\
\beta_{0} g_{0}(0)-\alpha_{0} g_{0}^{\prime}(0) & =1
\end{aligned}
$$

for $i=0$, and to the Cauchy problem

$$
\begin{aligned}
g_{i}^{\prime \prime} & =\rho g_{i-1}, \quad x \in(0,1) \\
g_{i}(0) & =0, \\
g_{i}^{\prime}(0) & =0
\end{aligned}
$$

for $i \geq 1$. Expanding $u$ on generating functions as in (1.16) rather than on powers of $x$ as in $[\mathbf{1 8 , 2 1 ]}$ was introduced in [17] and studied in [16].

The fact that the generating function $g_{i}$ is defined as the solution of a Cauchy problem, rather than the solution of a boundary-value problem, is crucial in the analysis developed here. First, it allows to prove that every initial state in the space $L_{\rho}^{1}$ (and not only states in some restricted class of Gevrey functions) can be driven to 0 in time $T$. Secondly, from (1.23)-(1.25), we see by an easy induction on $i$ that for $\rho \in L^{\infty}(0,1)$, the function $g_{i}$ is uniformly bounded by $C /(2 i)$ !, and hence the series in $(1.16)$ is indeed convergent when $y \in G^{s}([\tau, T])$ with $1<s<2$. 
The corresponding control function $h$ is given explicitly as

$$
h(t)= \begin{cases}0 & \text { if } 0 \leq t \leq \tau \\ \sum_{i \geq 0} y^{(i)}(t)\left(\alpha_{1} g_{i}(1)+\beta_{1} g_{i}^{\prime}(1)\right) & \text { if } \tau<t \leq T .\end{cases}
$$

It is easy to see that the function $u(x, t)$ defined in (1.16) satisfies (formally) (1.11), and also the condition $u(x, T)=0$ if $y^{(i)}(T)=0$ for all $i \in \mathbb{N}$, so that the null controllability can be established for some initial states. The main issue is then to extend it to every initial state $u_{0} \in L_{\rho}^{1}$. Following $[\mathbf{2 1}, \mathbf{2 2}, \mathbf{2 4}]$, we first use the strong smoothing effect of the heat equation to smooth out the state function in the time interval $(0, \tau)$. Next, to ensure that the two expressions of $u$ given in (1.15)-(1.16) coincide at $t=\tau$, we have to relate the eigenfunctions $e_{n}$ to the generating functions $g_{i}$.

It will be shown that any eigenfunction $e_{n}$ can be expanded in terms of the generating functions $g_{i}$ as

$$
e_{n}(x)=\zeta_{n} \sum_{i \geq 0}\left(-\lambda_{n}\right)^{i} g_{i}(x)
$$

with $\zeta_{n} \in \mathbb{R}$. Note that, for $\rho \equiv 1$ and $\left(\alpha_{0}, \beta_{0}, \alpha_{1}, \beta_{1}\right)=(0,1,0,1), \lambda_{n}=(n \pi)^{2}$ for all $n \geq 0, e_{0}(x)=1$ and $e_{n}(x)=\sqrt{2} \cos (n \pi x)$ for $n \geq 0$ while $g_{i}(x)=x^{2 i} /(2 i)$ !, so that (1.26) for $n \geq 1$ is nothing but the classical Taylor series expansion of $\cos (n \pi x)$ at $x=0$ :

$$
\cos (n \pi x)=\sum_{i \geq 0}(-1)^{i} \frac{(n \pi x)^{2 i}}{(2 i) !}
$$

Thus (1.26) can be seen as a natural extension of (1.27), in which the generating functions $g_{i}$, a priori not smoother than $W^{2, p}(0,1)$, replace the functions $x^{2 i} /(2 i)$ !.

The condition (1.8) is used to prove the estimate

$$
\left|g_{i}(x)\right| \leq \frac{C}{R^{2 i}(i !)^{2-\frac{1}{p}}}
$$

needed to ensure the convergence of the series in (1.16) when $y \in G^{s}([\tau, T])$ with $1<s<2-1 / p$.

Theorem 1.1 applies in particular to any system

$$
\begin{aligned}
\left(a(x) u_{x}\right)_{x}-u_{t} & =0, \quad x \in(0,1), t \in(0, T), \\
\alpha_{0} u(0, t)+\beta_{0}\left(a u_{x}\right)(0, t) & =0, \quad t \in(0, T), \\
\alpha_{1} u(1, t)+\beta_{1}\left(a u_{x}\right)(1, t) & =h(t), \quad t \in(0, T), \\
u(x, 0) & =u_{0}(x), \quad x \in(0,1),
\end{aligned}
$$

where $a(x)>0$ for a.e. $x \in(0,1)$ and $a+1 / a \in L^{1}(0,1)$. This includes the case where $a$ is measurable, positive and essentially bounded together with its inverse (but not necessarily piecewise continuous), and the case where $a(x)=x^{r}$ with $-1<r<1$. (Actually any $r \leq-1$ is also admissible, by picking $p>1$ sufficiently close to 1 in (1.8).) Note that our result applies as well to $a(x)=(1-x)^{r}$ with $0<r<1$, yielding a positive null controllability result when the control is applied at the point $(x=1)$ where the diffusion coefficient degenerates (see [5, Section 2.7]). Note also that the coefficient $a(x)$ is allowed to be degenerate/singular at a sequence of points: consider e.g. $a(x):=\left|\sin \left(x^{-1}\right)\right|^{r}$ with $-1<r<1$. Then $a+1 / a \in L^{1}(0,1)$.

The null controllability of (1.28)-(1.31) for $a(x)=x^{r}$ with $0<r<2$ was established (in appropriate spaces) in $[\mathbf{5}]$. 
Another important family of heat equations with variable coefficients is those with inverse square potential localized at the boundary, namely

$$
\begin{aligned}
u_{x x}+\frac{\mu}{x^{2}} u-u_{t} & =0, \quad x \in(0,1), t \in(0, T), \\
u(0, t) & =0, \quad t \in(0, T), \\
\alpha_{1} u(1, t)+\beta_{1} u_{x}(1, t) & =h(t), \quad t \in(0, T), \\
u(x, 0) & =u_{0}(x), \quad x \in(0,1),
\end{aligned}
$$

where $\mu \in \mathbb{R}$ is a given number. Note that Theorem 1.1 cannot be applied to (1.32)-(1.35), for $c(x)=\mu x^{-2}$ is not integrable on $(0,1)$. It was proved in [8] that $(1.32)-(1.35)$ is null controllable in $L^{2}(0,1)$ when $\mu \leq 1 / 4$ by combining Carleman inequalities to Hardy inequalities. We note that this result can be retrieved by the flatness approach as well.

Theorem 1.2. - Let $\mu \in(0,1 / 4],\left(\alpha_{1}, \beta_{1}\right) \in \mathbb{R}^{2} \backslash\{(0,0)\}, T>0$, and $\tau \in(0, T)$. Pick any $u_{0} \in L^{2}(0,1)$ and any $s \in(1,2)$. Then there exists a function $h \in G^{s}([0, T])$ with $h(t)=0$ for $0 \leq t \leq \tau$ and such that the solution $u$ of $(1.32)-(1.35)$ satisfies $u(T,)=$.0 . Moreover, $u \in G^{s}\left([\varepsilon, T], W^{1,1}(0,1)\right)$ for all $\varepsilon \in(0, T)$. Finally, if $0 \leq \mu<1 / 4$ and $r>(1+\sqrt{1-4 \mu}) / 2$, then $x^{r} u_{x} \in G^{s}\left([\varepsilon, T], W^{1,1}(0,1)\right)$ for all $\varepsilon \in(0, T)$.

The note is organized as follows. In Section 2 we give a sketch of the proof of Theorem 1.1. Section 3 is concerned with the numerical control of a heat equation with discontinuous coefficients, that may serve as a model for the heat conduction of a one-dimensional rod with constant thermals properties.

\section{Sketch of the proof of Theorem 1.1}

2.1. Reduction to the canonical form (1.11)-(1.14). — Let $a, b, c, \rho$, and $p$ be as in (1.5)-(1.8). Set

$$
\begin{aligned}
B(x) & :=\int_{0}^{x} \frac{b(s)}{a(s)} d s, \\
\tilde{a}(x) & :=a(x) e^{B(x)} \\
\tilde{c}(x) & :=(K \rho(x)-c(x)) e^{B(x)} .
\end{aligned}
$$

Then $B \in W^{1,1}(0,1), \tilde{c} \in L^{1}(0,1)$, and

$$
\tilde{a}(x)>0 \text { and } \tilde{c}(x) \geq 0 \text { for a.e. } x \in(0,1) .
$$

We introduce the solution $v$ to the elliptic boundary value problem

$$
\begin{aligned}
-\left(\tilde{a} v_{x}\right)_{x}+\tilde{c} v & =0, \quad x \in(0,1), \\
v(0)=v(1) & =1,
\end{aligned}
$$

and set

Finally, let

$$
u_{1}(x, t):=e^{-K t} u(x, t), \quad u_{2}(x, t):=\frac{u_{1}(x, t)}{v(x)}
$$

$$
L:=\int_{0}^{1}\left(a(s) v^{2}(s) e^{B(s)}\right)^{-1} d s, \quad y(x):=\frac{1}{L} \int_{0}^{x}\left(a(s) v^{2}(s) e^{B(s)}\right)^{-1} d s
$$

and

$$
\hat{u}(y, t):=u_{2}(x, t), \quad \hat{\rho}(y):=L^{2} a(x) v^{4}(x) e^{2 B(x)} \rho(x)
$$

for $0<t<1, y=y(x)$ with $0<x<1$. Then the following result holds. 
Proposition 2.1. - (i) $v \in W^{1,1}(0,1)$ and $0<v(x) \leq 1$ for all $x \in[0,1]$;

(ii) $y:[0,1] \rightarrow[0,1]$ is an increasing bijection with $y, y^{-1} \in W^{1,1}(0,1)$;

(iii) $\hat{\rho}(y)>0$ for a.e. $y \in(0,1)$, and $\hat{\rho} \in L^{p}(0,1)$;

(iv) $\hat{u}$ solves the system

$$
\begin{aligned}
\hat{u}_{y y}-\hat{\rho} \hat{u}_{t} & =0, \quad y \in(0,1), t \in(0, T), \\
\hat{\alpha}_{0} \hat{u}(0, t)+\hat{\beta}_{0} \hat{u}_{y}(0, t) & =0, \quad t \in(0, T), \\
\hat{\alpha}_{1} \hat{u}(1, t)+\hat{\beta}_{1} \hat{u}_{y}(1, t) & =\hat{h}(t):=e^{-K t} h(t), \quad t \in(0, T), \\
\hat{u}(y(x), 0) & =\frac{u_{0}(x)}{v(x)}, \quad x \in(0,1),
\end{aligned}
$$

for some $\left(\hat{\alpha}_{0}, \hat{\beta}_{0}\right),\left(\hat{\alpha}_{1}, \hat{\beta}_{1}\right) \in \mathbb{R}^{2} \backslash\{(0,0)\}$.

Proof: (i) Let $l:=\int_{0}^{1} d s / \tilde{a}(s)$ and $z(x):=l^{-1} \int_{0}^{x} d s / \tilde{a}(s)$. Then, one can see that $z$ is an increasing continuous bijection from $[0,1]$ to $[0,1]$, and (using a result due to Zareckii, see e.g. [3]) that $z^{-1} \in$ $W^{1,1}(0,1)$. Introduce $w(z):=v(x(z))$, which solves the boundary value problem

$$
\begin{aligned}
-\frac{d^{2} w}{d z^{2}}+\left(l^{2} \tilde{a} \tilde{c}\right)(x(z)) w & =0, \quad z \in(0,1), \\
w(0)=w(1) & =1 .
\end{aligned}
$$

It can be seen that $0<w \leq 1$ on $[0,1]$. The other properties (ii), (iii), and (iv) follow by direct calculations. See $[\mathbf{2 7}]$ for more details.

2.2. Null controllability of the control problem (1.11)-(1.14). - Assume given $p \in(1, \infty], \rho \in$ $L^{p}(0,1)$ with $\rho(x)>0$ for a.e. $x \in(0,1)$, and $\left(\alpha_{0}, \beta_{0}\right),\left(\alpha_{1}, \beta_{1}\right) \in \mathbb{R}^{2} \backslash\{(0,0)\}$. Let ${ }^{\prime}=d / d x$, and let

$$
L_{\rho}^{2}:=\left\{f:(0,1) \rightarrow \mathbb{R} ;\|f\|_{L_{\rho}^{2}}^{2}:=\int_{0}^{1}|f(x)|^{2} \rho(x) d x<\infty\right\} .
$$

Then the following result holds.

Proposition 2.2. - Let $p, \rho, \alpha_{0}, \beta_{0}, \alpha_{1}$, and $\beta_{1}$ be as above. Then there are a sequence $\left(e_{n}\right)_{n \geq 0}$ in $L_{\rho}^{2}$ and a sequence $\left(\lambda_{n}\right)_{n \geq 0}$ in $\mathbb{R}$ such that

(i) $\left(e_{n}\right)_{n \geq 0}$ is an orthonormal basis in $L_{\rho}^{2}$;

(ii) For all $n \geq 0, e_{n} \in W^{2, p}(0,1)$ and $e_{n}$ solves

$$
\begin{aligned}
-e_{n}^{\prime \prime} & =\lambda_{n} \rho e_{n} \quad \text { in }(0,1), \\
\alpha_{0} e_{n}(0)+\beta_{0} e_{n}^{\prime}(0) & =0 \\
\alpha_{1} e_{n}(1)+\beta_{1} e_{n}^{\prime}(1) & =0 .
\end{aligned}
$$

(iii) The sequence $\left(\lambda_{n}\right)_{n \geq 0}$ is strictly increasing, and for some constant $C>0$

$$
\lambda_{n} \geq C n \quad \text { for } n \gg 1 \text {. }
$$

Proof: Introduce for $\lambda^{*} \gg 1$ the boundary-value problem

$$
\begin{aligned}
-u^{\prime \prime}+\lambda^{*} \rho u=\rho f & \quad \text { in }(0,1), \\
\alpha_{0} u(0)+\beta_{0} u^{\prime}(0) & =0 \\
\alpha_{1} u(1)+\beta_{1} u^{\prime}(1) & =0 .
\end{aligned}
$$


Then, introducing a variational formulation and applying Lax-Milgram theorem, we obtain the existence and uniqueness of a solution $u \in W^{2,1}(0,1)$ of $(2.13)$-(2.15) for any $f \in L_{\rho}^{2}$. The results in (i)-(ii) follow from an application of the spectral theorem. Finally, (iii) is established by using Prüfer substitution. We refer the reader to $[\mathbf{2 7}]$ for more details.

We now turn our attention to the generating functions $g_{i}(i \geq 0)$ defined along (1.20)-(1.25).

Proposition 2.3. -

(i) $g_{0}(x)=\left(\alpha_{0}^{2}+\beta_{0}^{2}\right)^{-1}\left(\beta_{0}-\alpha_{0} x\right)$

(ii) There are some constants $C, R>0$ such that

$$
\left\|g_{i}\right\|_{W^{2, p}(0,1)} \leq \frac{C}{R^{i}(i !)^{2-\frac{1}{p}}} \quad \forall i \geq 0 .
$$

Proof: (i) is obvious, and (ii) is obtained inductively from the formula

$$
g_{i}(x)=\int_{0}^{x}\left(\int_{0}^{s} \rho(\sigma) g_{i-1}(\sigma) d \sigma\right) d s .
$$

See $[\mathbf{2 7}]$ for more details.

The fact that we can expand the eigenfunctions in terms of the generating functions is detailed in the following

Proposition 2.4. - There is some sequence $\left(\zeta_{n}\right)_{n \geq 0}$ of real numbers such that for all $n \geq 0$

$$
e_{n}=\zeta_{n} \sum_{i \geq 0}\left(-\lambda_{n}\right)^{i} g_{i} \quad \text { in } W^{2, p}(0,1)
$$

Furthermore, for some constant $C>0$, we have

$$
\left|\zeta_{n}\right| \leq C\left(1+\left|\lambda_{n}\right|^{\frac{3}{2}}\right) \quad \forall n \geq 0 .
$$

Proof: Let $\tilde{e}:=\zeta_{n} \sum_{i \geq 0}\left(-\lambda_{n}\right)^{i} g_{i}$, where $\zeta_{n} \in \mathbb{R}$. Then we infer from (1.20)-(1.25) that $\tilde{e}^{\prime \prime}=-\lambda_{n} \rho \tilde{e}$ and that

$$
\alpha_{0} \tilde{e}(0)+\beta_{0} \tilde{e}^{\prime}(0)=0
$$

If we pick $\zeta_{n}:=\beta_{0} e_{n}(0)-\alpha_{0} e_{n}^{\prime}(0)$, then we easily see that $e_{n}=\tilde{e}$. The estimate $(2.18)$ is proved by direct calculations. We refer the reader to $[\mathbf{2 7}]$ for more details.

Since $p>1$, for any $s \in\left(1,2-\frac{1}{p}\right)$ and any $0<\tau<T$, one may pick a function $\varphi \in G^{s}([0,2 T])$ such that

$$
\varphi(t)= \begin{cases}1 & \text { if } t \leq \tau \\ 0 & \text { if } t \geq T\end{cases}
$$

We are in a position to derive the null controllability of (1.11)-(1.14). Let $u_{0} \in L_{\rho}^{2}$. Since $\left(e_{n}\right)_{n \geq 0}$ is an orthonormal basis in $L_{\rho}^{2}$, we can write

$$
u_{0}=\sum_{n \geq 0} c_{n} e_{n} \quad \text { in } L_{\rho}^{2}
$$

with $\sum_{n \geq 0}\left|c_{n}\right|^{2}<\infty$. Let

$$
y(t):=\varphi(t) \sum_{n \geq 0} c_{n} \zeta_{n} e^{-\lambda_{n} t} \quad \text { for } t \in[\tau, T]
$$


and

$$
u(x, t)= \begin{cases}\sum_{n \geq 0} c_{n} e^{-\lambda_{n} t} e_{n}(x) & \text { if } 0 \leq t \leq \tau \\ \sum_{i \geq 0} y^{(i)}(t) g_{i}(x) & \text { if } \tau<t \leq T\end{cases}
$$

The main result in this section is the following

Theorem 2.5. - Let $p \in(1, \infty], \rho \in L^{p}(0,1)$ with $\rho(x)>0$ for a.e. $x \in(0,1), T>0, \tau \in(0, T)$, and $\left(\alpha_{0}, \beta_{0}\right),\left(\alpha_{1}, \beta_{1}\right) \in \mathbb{R}^{2} \backslash\{(0,0)\}$. Let $u_{0} \in L_{\rho}^{2}$ be decomposed as in $(2.19)$, and let $y$ be as in $(2.20)$. Then $y \in G^{s}([\tau, T])$, and the control

$$
h(t)= \begin{cases}0 & \text { if } 0 \leq t \leq \tau, \\ \sum_{i \geq 0} y^{(i)}(t)\left(\alpha_{1} g_{i}(1)+\beta_{1} g_{i}^{\prime}(1)\right) & \text { if } \tau<t \leq T .\end{cases}
$$

is such that the solution $u$ of (1.11)-(1.14) satisfies $u(., T)=0$. Moreover $u$ is given by $(2.21), h \in$ $G^{s}([0, T])$, and $u \in C\left([0, T], L_{\rho}^{2}\right) \cap G^{s}\left([\varepsilon, T], W^{2, p}(0,1)\right)$ for all $0<\varepsilon \leq T$.

Proof: It is clear that the function $u$ solves formally $(1.11)-(1.14)$ on $(0, \tau)$ and on $(\tau, T)$, together with $u(., T)=0$. The main concern is thus the convergence of the two series in (2.21) and the fact that they coincide at $t=\tau$. See $[\mathbf{2 7}]$ for the details.

Theorem 1.1 follows from Theorem 2.5. Modifying slightly the first step in the proof of Theorem 1.1, we can reduce (1.32)-(1.35) to the canonical form (1.11)-(1.14), so that the conclusion of Theorem 1.2 follows from Theorem 2.5.

As a possible application, we consider the boundary control by the flatness approach of radial solutions of the heat equation in the ball $B(0,1) \subset \mathbb{R}^{N}(2 \leq N \leq 3)$. Using the radial coordinate $r=|x|$, we thus consider the system

$$
\begin{aligned}
u_{r r}+\frac{N-1}{r} u_{r}-u_{t} & =0, \quad r \in(0,1), t \in(0, T), \\
u_{r}(0, t) & =0, \quad t \in(0, T), \\
\alpha_{1} u(1, t)+\beta_{1} u_{r}(1, t) & =h(t), \quad t \in(0, T) \\
u(r, 0) & =u_{0}(r), \quad r \in(0,1) .
\end{aligned}
$$

Note that Theorem 1.1 cannot be applied directly to (2.23)-(2.26), for (1.7) fails. (Note that, in sharp contrast, the control on a ring-shaped domain $\left\{r_{0}<|x|<r_{1}\right\}$ with $r_{1}>r_{0}>0$ is fully covered by Theorem 1.1, the coefficients in (2.23) being then smooth and bounded.)

We use the following change of variables from $[\mathbf{9}]$ which allows to remove the term with the first order derivative in $r$ in $(2.23)$ :

$$
u(r, t)=\tilde{u}(r, t) \exp \left(-\frac{1}{2} \int_{0}^{r} \frac{N-1}{s} d s\right)=\frac{\tilde{u}(r, t)}{r^{\frac{N-1}{2}}} .
$$

Then (2.23) becomes

$$
\tilde{u}_{r r}+\frac{(N-1)(3-N)}{4} \frac{\tilde{u}}{r^{2}}-\tilde{u}_{t}=0 .
$$

This equation has to be supplemented with the boundary/initial conditions

$$
\begin{aligned}
\tilde{u}(0, t) & =0, \quad t \in(0, T), \\
\left(\alpha_{1}-\frac{N-1}{2} \beta_{1}\right) \tilde{u}(1, t)+\beta_{1} \tilde{u}_{r}(1, t) & =h(t), \quad t \in(0, T), \\
\tilde{u}(r, 0) & =r^{\frac{N-1}{2}} u_{0}(r), \quad r \in(0, R) .
\end{aligned}
$$


For $N=3,(2.28)$ reduces to the simple heat equation $\tilde{u}_{r r}-\tilde{u}_{t}=0$ to which Theorem 1.1 can be applied.

For $N=2,(2.28)-(2.31)$ is of the form (1.32)-(1.35) with $\mu=1 / 4$. Therefore Theorem 1.2 can be applied.

\section{Numerical control of the 1-D heat equation with discontinuous coefficients}

We consider the heat conduction in a one-dimensional rod made of two sections with constant thermal properties. Without restriction, we can assume the rod has length 1, with one section of length $X$ and the other of length $1-X$. The evolution of the temperature $u$ is given by the heat equation

$$
\rho \theta_{t}(x, t)=\left(a \theta_{x}\right)_{x}(x, t) .
$$

$a$ and $\rho$ are the piecewise constant functions on $(0,1)$ :

$$
(a(x), \rho(x)):= \begin{cases}\left(a_{0}, \rho_{0}\right), & 0<x<X \\ \left(a_{1}, \rho_{1}\right), & X<x<1\end{cases}
$$

where $a_{0}, a_{1}, \rho_{0}$ and $\rho_{1}$ are strictly positive constants.

At the 0 -end, the rod is submitted to the constant ambient temperature $\theta_{0}$, and at the 1 -end to a time-varying heat source (the control input) of temperature $\theta_{1}(t)$. The heat flux $-a \theta_{x}$ at the ends obeys the convection conditions

$$
\begin{aligned}
-\left(a \theta_{x}\right)(0, t) & =h_{0}\left(\theta_{0}-\theta(0, t)\right), \\
-\left(a \theta_{x}\right)(1, t) & =h_{1}\left(\theta(1, t)-\theta_{1}(t)\right)
\end{aligned}
$$

with $h_{0}, h_{1}$ some positive constants. Setting $u(x, t):=\theta(x, t)-\theta_{0}$ and taking as input $h(t):=\theta_{1}(t)-\theta_{0}$ results in the boundary value problem

$$
\begin{aligned}
\left(a(x) u_{x}\right)_{x}-\rho(x) u_{t} & =0, \quad x \in(0,1), t \in(0, T), \\
\alpha_{0} u(0, t)+\beta_{0}\left(a u_{x}\right)(0, t) & =0, \quad t \in(0, T), \\
\alpha_{1} u(1, t)+\beta_{1}\left(a u_{x}\right)(1, t) & =h(t), \quad t \in(0, T), \\
u(x, 0) & =u_{0}(x), \quad x \in(0,1),
\end{aligned}
$$

where the constants $\alpha_{0}, \beta_{0}, \alpha_{1}, \beta_{1}$ satisfy $\alpha_{0}^{2}+\beta_{0}^{2}>0, \alpha_{1}^{2}+\beta_{1}^{2}>0, \alpha_{0} \beta_{0} \leq 0$ and $\alpha_{1} \beta_{1} \geq 0$. Notice the two limiting cases: $\beta_{i}=0$ (Dirichlet conditions), obtained when taking $h_{i} \rightarrow \infty ; \alpha_{i}=0$ (Neumann conditions), obtained when taking as control input $h(t):=h_{1}\left(\theta_{1}(t)-\theta(1, t)\right)$ and letting $h_{0}=0$.

Included in the formulation of the system is the fact that a solution $u$ and its quasi-derivative $a u_{x}$ are continuous on $[0,1]$, and in particular at $x=X$ (whereas $u_{x}$ will in general be discontinuous at $X$ ). We could thus rewrite (3.1) more explicitly as the piecewise constant heat equation

$$
\left\{\begin{array}{l}
u_{t}(x, t)=\frac{a_{0}}{\rho_{0}} u_{x x}(x, t), \quad 0<x<X, \\
u_{t}(x, t)=\frac{a_{1}}{\rho_{1}} u_{x x}(x, t), \quad X<x<1
\end{array}\right.
$$

together with the so-called interface conditions

$$
\begin{aligned}
u\left(X^{-}, t\right) & =u\left(X^{+}, t\right), \\
a_{0} u_{x}\left(X^{-}, t\right) & =a_{1} u_{x}\left(X^{+}, t\right) .
\end{aligned}
$$

From the flatness approach developed in the previous sections, we know that the trajectory $u$ of (3.1)-(3.4) is given explicitly in (1.15)-(1.16), the eigenfunctions $e_{n}$ 's being as in (1.17)-(1.19), and the generating functions $g_{i}$ 's being as in (1.20)-(1.25).

We refer the reader to $[\mathbf{2 5}]$ for the details concerning the numerical computation of the $e_{n}$ 's and the $g_{i}$ 's. 
The control input in the time interval $(\tau, T)$ reads

$$
h(t):=\sum_{i \geq 0}\left(\alpha_{1} g_{i}(1)+\beta_{1} g_{i}^{\prime}(1)\right) y^{(i)}(t)
$$

where

$$
y(t):=\phi_{s}\left(\frac{t-\tau}{T-\tau}\right) \sum_{n \geq 0} c_{n} \zeta_{n} e^{-\lambda_{n} t}
$$

and the $c_{n}$ 's denote the Fourier coefficients of the initial state decomposed along the $e_{n}$ 's:

$$
u_{0}(x):=\sum_{n \geq 0} c_{n} e_{n}(x) .
$$

The function $\phi_{s}$ in (3.6) is the Gevrey "step function"

$$
\phi_{s}(t):= \begin{cases}1 & \text { if } t \leq 0, \\ 0 & \text { if } t \geq 1, \\ 1-\frac{\int_{0}^{t} \varphi_{s}(z) d z}{\int_{0}^{1} \varphi_{s}(z) d z} & \text { if } t \in(0,1)\end{cases}
$$

where the "bump function" $\varphi_{s}$ is defined as

$$
\varphi_{s}(t)= \begin{cases}0 & \text { if } t \notin(0,1) \\ \exp \left(\frac{-1}{M t^{k}(1-t)^{k}}\right) & \text { if } t \in(0,1)\end{cases}
$$

with $k=(s-1)^{-1}>1$ and $M>0$ some constants. It is well known that both $\varphi_{s}$ and $\phi_{s}$ are Gevrey of order $s$, with $s \in(1,2)$.

A practical problem when implementing the control (3.6) is to evaluate sufficiently many derivatives of $\phi_{s}$, i.e. of $\varphi_{s}$. Numerical computations (with e.g. finite differences) or symbolic computations cannot be used in practice to evaluate more than 20 derivatives. Nevertheless, much more derivatives can be computed with accuracy by proceeding inductively. We first note that

$$
p^{k+1} \dot{\varphi}_{s}=k \dot{p} \varphi_{s}
$$

where $p(t):=M^{\frac{1}{k}} t(1-t)$ is a polynomial function of degree 2 (hence its derivatives of order $>2$ are zero).

Derivating $i$ times in (3.7) and using Leibniz' rule results in

$$
p^{k+1} \varphi_{s}^{(i+1)}+\sum_{j=1}^{i}\left(\begin{array}{l}
i \\
j
\end{array}\right)\left(p^{k+1}\right)^{(j)} \varphi_{s}^{(i+1-j)}=k\left(\dot{p} \varphi_{s}^{(i)}+i \ddot{p} \varphi_{s}^{(i-1)}\right)
$$

This formula gives $\varphi_{s}^{(i+1)}$ in terms of $\varphi_{s}^{(0)}, \ldots, \varphi_{s}^{(i)}$, and the derivatives of $P:=p^{k+1}$ that can be obtained similarly, by applying Leibniz' rule to both sides of

$$
p \dot{P}=(k+1) \dot{p} P \text {. }
$$

Note that, in order to avoid computing ratios of very large numbers, it is better in practice to use recursion formulas for $\tilde{\varphi}_{s}^{(i)}:=\varphi_{s}^{(i)} /(2 i)$ ! and for $\tilde{P}^{(i)}:=P^{(i)} / i$ ! (see [25].)

Using this procedure, about 140 derivatives can be efficiently determined with Matlab double-precision arithmetics.

We conclude this note with some numerical simulation. We use as parameters: $X=1 / 2, a_{0}=10 / 19$, $\rho_{0}=15 / 8, a_{1}=10, \rho_{1}=1 / 8, \alpha_{0}=\cos (\pi / 3), \beta_{0}=-\sin (\pi / 3), \alpha_{1}=\cos (\pi / 4), \beta_{1}=\sin (\pi / 4)$. We take as initial condition $u_{0}(x):=\frac{1}{2} 1_{(1 / 2,1)}(x)-\frac{1}{2} 1_{(0,1 / 2)}(x)$, and as control times: $\tau=0.05$ and $T=0.35$. We pick also $s=1.65$ and $M=2$. The series for $h$ and $y$ in (3.5)-(3.6) were truncated at a "large enough" 
order for a good accuracy, namely $\bar{i}=130$ and $\bar{n}=60$; a fairly large $\bar{i}$ is needed here because $(T-\tau) a_{0} / \rho_{0}$ is rather small. Figure 1 shows the resulting temperature $u$; the discontinuity of $u_{x}$ at $x=X$ is clearly visible. We refer the interested reader to $[\mathbf{2 5}]$ for more details about the numerical investigation of the

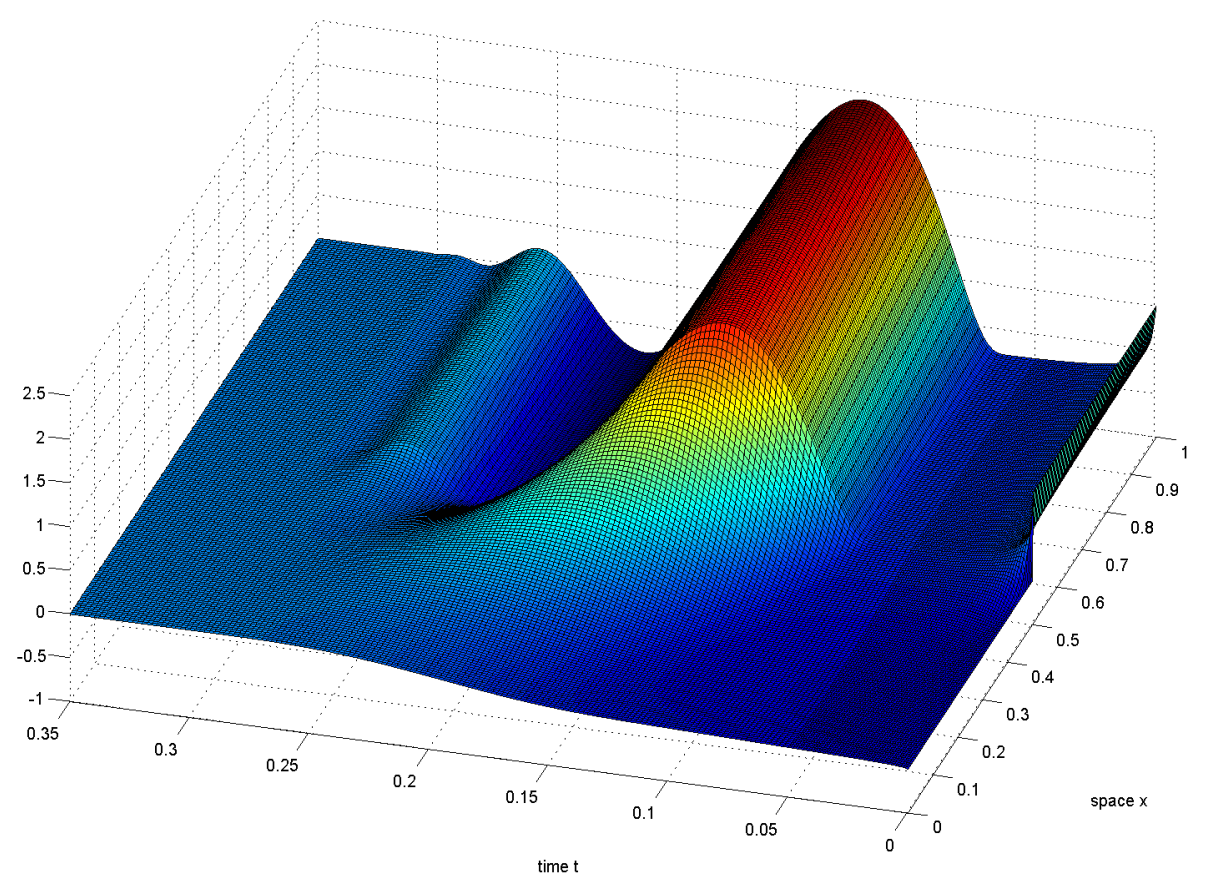

Figure 1. Evolution of the temperature $u(x, t)$

control problem (3.1)-(3.4).

\section{Numerical control of a degenerate 1-D heat equation}

We now apply the approach of the previous section to the degenerate heat equation

$$
\begin{aligned}
& \theta_{t}(x, t)-\left(x^{\gamma} \theta_{x}(x, t)\right)_{x}=0,(x, t) \in(0,1) \times(0, T) \\
& \alpha_{0} \theta(0, t)+\left.\beta_{0}\left(x^{\gamma} \theta_{x}(x, t)\right)\right|_{x=0}=u(t), \\
& \alpha_{1} \theta(1, t)+\beta_{1} \theta_{x}(1, t)=0,
\end{aligned}
$$

with $0 \leq \gamma<1$. The controllability properties of this system (with Dirichlet boundary conditions) are studied in [7]; in particular, it is shown in [7] that the cost in terms of the $H^{1}(0, T)$-norm of the control achieving null controllability blows up as $\gamma$ tends to $1^{-}$. 

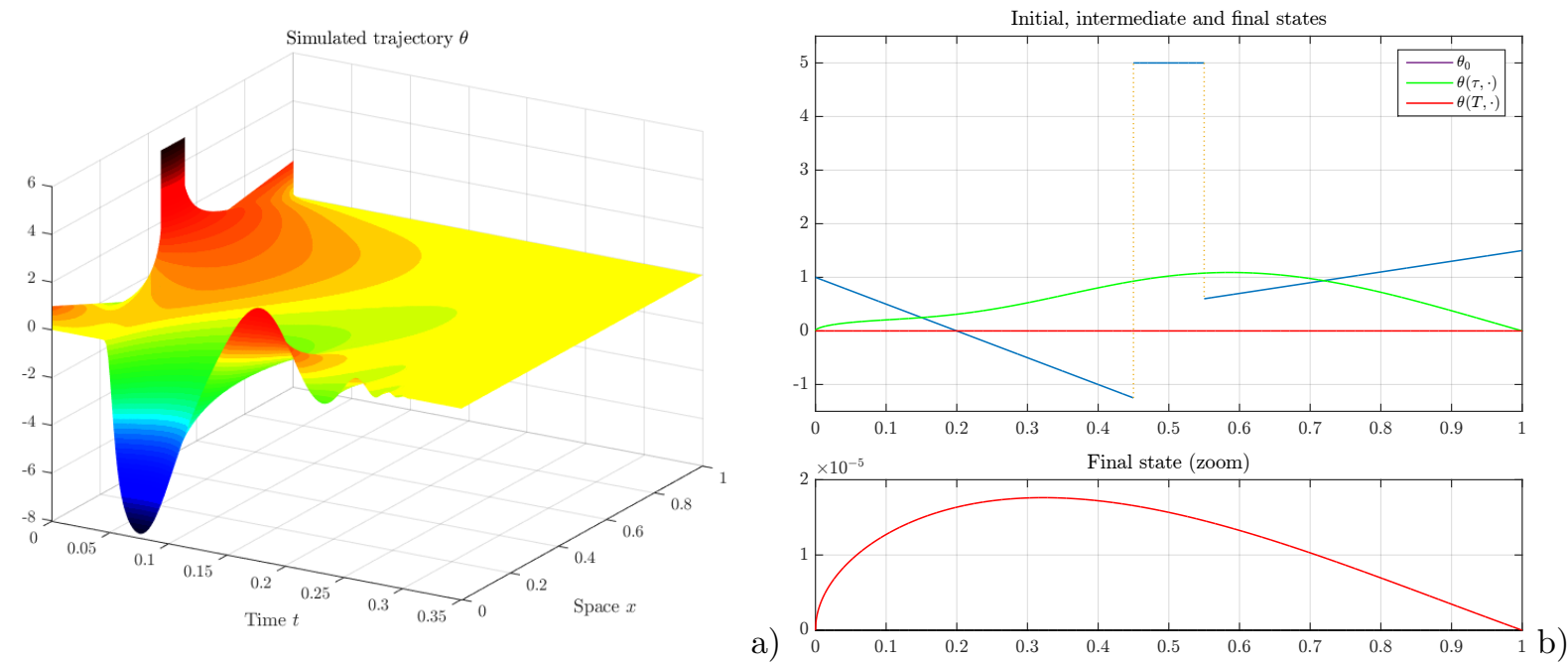

Figure 2. a) Evolution of $\theta(\gamma=1 / 2)$;

b) corresponding initial, intermediate, and final states.

The assumptions (1.7)-(1.8) are clearly satisfied, with $K=0$ and $p=\infty$. The system is put into its canonical form by setting $\tilde{x}:=x^{1-\gamma}$ and $\tilde{\theta}(\tilde{x}, t):=\theta(x, t)$, which immediately yields

$$
\begin{gathered}
(1-\gamma)^{-2} \tilde{x}^{\frac{\gamma}{1-\gamma}} \tilde{\theta}_{t}(\tilde{x}, t)-\tilde{\theta}_{\tilde{x} \tilde{x}}(\tilde{x}, t)=0, \quad(\tilde{x}, t) \in(0,1) \times(0, T) \\
\alpha_{0} \tilde{\theta}(0, t)+(1-\gamma) \beta_{0} \tilde{\theta}_{\tilde{x}}(0, t)=u(t), \\
\alpha_{1} \tilde{\theta}(1, t)+(1-\gamma) \beta_{1} \tilde{\theta}_{\tilde{x}}(1, t)=0 .
\end{gathered}
$$

For the actual scenario $(\gamma=0.5, T=0.35, \tau=T / 10, s=1.7)$, Neumann boundary conditions are used $\left(\alpha_{0}=\alpha_{1}=0, \beta_{0}=\beta_{1}=1\right)$. The initial condition is the piecewise linear function $\theta_{0}(x):=1-5 x, x \in$ $(0,0.45), \theta_{0}(x):=5.5, x \in(0.45,0.55)$, and $\theta_{0}(x):=2 x-0.5, x \in(0.55,1)$.

We refer the interested reader to $[\mathbf{2 6}]$ for the details about the numerical scheme. 


\section{References}

[1] G. Alessandrini and L. Escauriaza. Null-controllability of one-dimensional parabolic equations. ESAIM Control Optim. Calc. Var., 14(2):284-293, 2008.

[2] A. Benabdallah, Y. Dermenjian, and J. Le Rousseau. Carleman estimates for the one-dimensional heat equation with a discontinuous coefficient and applications to controllability and an inverse problem. J. Math. Anal. Appl., 336(2):865-887, 2007.

[3] V. I. Bogachev. Measure Theory. Springer-Verlag, Berlin, 2007.

[4] P. Cannarsa, P. Martinez, and J. Vancostenoble. Persistent regional null controllability for a class of degenerate parabolic equations. Commun. Pure Appl. Anal., 3(4):607-635, 2004.

[5] P. Cannarsa, P. Martinez, and J. Vancostenoble. Carleman estimates for a class of degenerate parabolic operators. SIAM J. Control Optim., 47(1):1-19, 2008.

[6] P. Cannarsa, P. Martinez, and J. Vancostenoble. Carleman estimates and null controllability for boundarydegenerate parabolic operators. C. R. Math. Acad. Sci. Paris, 347(3-4):147-152, 2009.

[7] P. Cannarsa, P. Martinez, and J. Vancostenoble. The cost of controlling degenerate parabolic equations by boundary controls. ArXiv e-prints, arXiv:1511.06857, 2015.

[8] C. Cazacu. Controllability of the heat equation with an inverse-square potential localized on the boundary. SIAM J. Control Optim., to appear.

[9] D. Colton. Integral operators and reflection principles for parabolic equations in one space variable. J. Differential Equations, 15:551-559, 1974.

[10] S. Ervedoza. Control and stabilization properties for a singular heat equation with an inverse-square potential. Comm. Partial Differential Equations, 33(10-12):1996-2019, 2008.

[11] H. Fattorini and D. Russell. Exact controllability theorems for linear parabolic equations in one space dimension. Arch. Rational Mech. Anal., 43(4):272-292, 1971.

[12] C. Flores and L. de Teresa. Carleman estimates for degenerate parabolic equations with first order terms and applications. C. R. Math. Acad. Sci. Paris, 348(7-8):391-396, 2010.

[13] A. V. Fursikov and O. Y. Imanuvilov. Controllability of evolution equations, volume 34 of Lecture Notes Series. Seoul National University Research Institute of Mathematics Global Analysis Research Center, 1996.

[14] O. Y. Imanuvilov. Controllability of parabolic equations. Mat. Sb., 186(6):109-132, 1995.

[15] B. Jones Jr. A fundamental solution for the heat equation which is supported in a strip. J. Math. Anal. Appl., 60(2):314-324, 1977.

[16] B. Laroche. Extension de la notion de platitude à des systèmes décrits par des équations aux dérivées partielles linéaires. PhD thesis, Ecole des Mines de Paris, 2000.

[17] B. Laroche and P. Martin. Motion planning for a 1-D diffusion equation using a Brunovsky-like decomposition. In Proceedings of the International Symposium on the Mathematical Theory of Networks and Systems (MTNS), 2000.

[18] B. Laroche, P. Martin, and P. Rouchon. Motion planning for the heat equation. Int J Robust Nonlinear Control, 10(8):629-643, 2000.

[19] G. Lebeau and L. Robbiano. Contrôle exact de l'équation de la chaleur. Comm. Partial Differential Equations, 20(1-2):335-356, 1995.

[20] W. A. J. Luxemburg and J. Korevaar. Entire functions and Müntz-Szász type approximation. Trans. Amer. Math. Soc., 157:23-37, 1971.

[21] P. Martin, L. Rosier, and P. Rouchon. Null controllability of the 1D heat equation using flatness. In 1st IFAC workshop on Control of Systems Governed by Partial Differential Equations (CPDE2013), pages 7-12, 2013.

[22] P. Martin, L. Rosier, and P. Rouchon. Null controllability of the 2D heat equation using flatness. In 52nd IEEE Conference on Decision and Control (CDC 2013), pages 3738-3743, 2013.

[23] P. Martin, L. Rosier, and P. Rouchon. Controllability of the 1D schrödinger equation by the flatness approach. In 19th World Congress of the International Federation of Automatic Control (IFAC 2014), pages 646-651, 2014. 
[24] P. Martin, L. Rosier, and P. Rouchon. Null controllability of the heat equation using flatness. Automatica J. IFAC, 50(12):3067-3076, 2014.

[25] P. Martin, L. Rosier, and P. Rouchon. Null controllability using flatness: a case study of 1-D heat equation with discontinuous coefficients. In European Control Conference ECC15, 2015.

[26] P. Martin, L. Rosier, and P. Rouchon. Flatness and null controllability of 1-d parabolic equations. PAMM, 16(1):47-50, 2016.

[27] P. Martin, L. Rosier, and P. Rouchon. Null controllability of one-dimensional parabolic equations by the flatness approach. SIAM J. Control Optim., 54(1):198-220, 2016.

[28] P. Martin, L. Rosier, and P. Rouchon. On the reachable states for the boundary control of the heat equation. Appl. Math. Res. Express. AMRX, 2016(2):181-216, 2016.

[29] T. Meurer. Control of Higher-Dimensional PDEs: Flatness and Backstepping Designs. Communications and Control Engineering. Springer, 2012.

Philippe Martin, Centre Automatique et Systèmes, MINES ParisTech, PSL Research University, 60 boulevard SaintMichel, 75272 Paris Cedex 06, France • E-mail : philippe.martin@mines-paristech.fr

Lionel Rosier, Centre Automatique et Systèmes, MINES ParisTech, PSL Research University, 60 boulevard SaintMichel, 75272 Paris Cedex 06, France • E-mail : lionel.rosier@mines-paristech.fr

Pierre Rouchon, Centre Automatique et Systèmes, MINES ParisTech, PSL Research University, 60 boulevard SaintMichel, 75272 Paris Cedex 06, France • E-mail : pierre.rouchon@mines-paristech.fr 